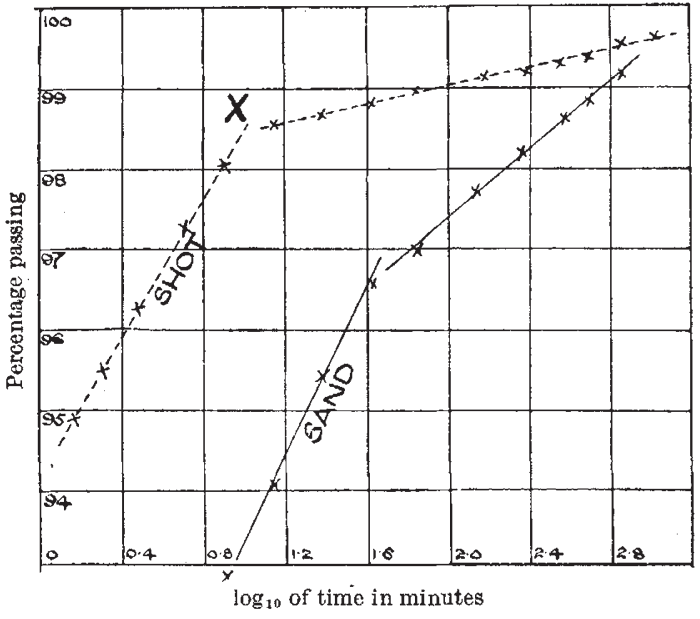

materials, or to suggest an explanation for the abrupt changes in the quantity-logarithmic time curves.

J. K. Alderman

Basil Standing

Whitworth Engineering Laboratories,

University, Manchester 13.

March 1.

'Krumbein and Pettijohn, "Manual of Sedimentary Petrology".

${ }^{2}$ Wentworth, Amer. J. Sci., 13, 399 (1927).

\section{Runs Up and Down on a Lattice}

$N$ different numbers arranged at random in a sequence can be considered to be consisting of $k$ groups of numbers alternately in ascending and descending order. Groups of numbers in ascending and descending order are called runs up and down respectively. Kermack and McKendrick ${ }^{1}$, Levene and Wolfowitz ${ }^{2}$ and others have dealt with the theory of runs up and down. Recently ${ }^{3}$, I developed independently a method similar to that of Fréchet ${ }^{4}$ for calculating the factorial moments of a large number of distributions considered in the statistical literature. This method is directly applicable for calculating the factorial and product moments of the distributions arising in the theory of runs up and down. Thus, for example, the second factorial moment for the dis. tribution of the total number of runs of length $p$, or of $p$ and more, is the sum of the expectations of the different configurations giving two such runs. The expectation for each of the configurations is the product of their probability and the number of configurations that can be had from the $N$ numbers. The probability for any of the configurations can be evaluated step by step by breaking it into two independent sections and expressing it as the sum of two new configurations as illustrated below.

Let $A$ represent the configuration for which the probability is to be determined.

$$
p / l^{p /}
$$

$A$ consists of two runs of length $p$ formed from $2 p$ numbers.

The probability for the above configuration is the product of the probabilities for two separate ascending runs each of length $p$ minus the probability of an ascending run of length $2 p$

$$
=\left\{\frac{1}{p ! p !}-\frac{1}{(2 p) !}\right\} \text {. }
$$

Similarly, the product of the probabilities for runs of length $(p-1)$ and $(q-1)$ is the sum of the probabilities for the configurations $B$ and $C$ noted below :
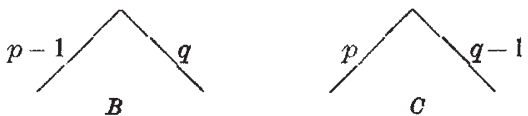

From the above relation, it can be shown that the probability for $B$ is $1 /(p+q-2)$. $(p-2) !(q-1)$ !

Using the above method, the expectation and the variance for the total number of peaks and troughs ${ }^{5}$, or the number of runs up and down, along the $n$ axes for $\prod_{1}^{n} l_{r}$ different numbers distributed in the form of an $n$-dimensional lattice of side $l_{1}, l_{2}, \ldots, l_{n}$ have been calculated, and are given below :

$$
\begin{aligned}
& \left.\begin{array}{l}
\text { Expected No. of } \\
\quad \text { troughs and peaks }
\end{array}\right\}=\frac{2}{3}\left\{n\left(1^{n}\right)-2\left(1^{n-1}\right)\right\},(1) \\
& \left.\begin{array}{l}
\text { Expected No. of } \\
\text { runs up and down }
\end{array}\right\}=\frac{1}{3}\left\{2 n\left(1^{n}\right)-\left(1^{n-1}\right)\right\} \text {, } \\
& \begin{array}{l}
\text { Variance for }(1) \text { and }\}=\frac{1}{90}\left\{16 n\left(1^{n}\right)-29\left(1^{n-i}\right)\right\},(3) \\
(2)
\end{array}
\end{aligned}
$$

where $\left(1^{n}\right)$ denotes the monomial symmetric function of degree $r$ in $l_{1}, l_{2}, \ldots l_{n}$. For $n=1$, (3) reduces to the expression given by Kendall ${ }^{5}$.

The higher cumulants are linear expressions in $\left(1^{n}\right),\left(1^{n-1}\right), \ldots$, and therefore the distribution approaches the normal form when $l_{1}, l_{2}, \ldots l_{n} \rightarrow \infty$.

Full details will be published in the Journal of the Indian Society of Agricultural Statistics.

Indian Council of Agricultural Research,

$$
\text { P. V. KRISHNa IYer }
$$

\section{New Delhi. \\ April 8.}

' Kermack, W. O., and McKendrick, M. G., Proc. Roy. Soc. Edin., 47 228 and 332 (1937).

${ }^{2}$ Levene, H., and Wolfowitz, J., Ann. Math. Stat., 13, 58 (1944).

${ }^{3}$ Krishna Iyer, P. V., Nature, 164, 282 (1948).

- Fréchet, M., "Les Probabilités Associées à un système d'événements, Compatibles et Dépendants" (Paris : Herman and Co., Ltd., 1940 and 1943).

sendall, M. G., "The Advanced Theory of Statistics", 2, 124 (London: Griffin and Co., Ltd., 1946).

\section{Mineralogical Composition of Quaternary Swedish Clays}

THE general opinion was that the quaternary, that is, glacial and postglacial, clays of Sweden consist mainly of physical weathering products from the ice age. During this age, the moving glaciers attacked the top of the rocks (mainly granite, gneiss and similar materials) and ground them into particles of various size : boulders, stones, gravel, sand, silt and clay. As a result of transportation by ice and glacial rivers and subsequent deposition, the different quaternary formations were formed, the clays being deposited in the sea. It was therefore logical to regard the clay fraction as rock 'flour', built up of primary minerals, typical for the original rocks but more or less chemically weathered. The micas were assumed to be responsible for the high plasticity of the clays. However, doubts arose later as to the correctness of the 